Abbreviated Key Title: Sch J Phys Math Stat

ISSN 2393-8056 (Print) | ISSN 2393-8064 (Online)

Journal homepage: https://saspublishers.com/sjpms/

\title{
Steady Generalized Plane Couette Flow of Viscous Incompressible Fluid between Two Porous Parallel Plates through Porous Medium with Magnetic Field
}

Dr. Anand Swrup Sharma (D.Sc. Scholar)

Professor, Dept. of Applied Sciences (Mathematics), Future University, Bareilly (U P), India

DOI: $10.36347 /$ sjpms.2020.v07i08.004

| Received: 10.08.2020| Accepted: 18.08.2020| Published: 20.08.2020

*Corresponding author: Dr. Anand Swrup Sharma

Email: sharma.as09@gmail.com

Abstract

Review Article

In this paper, we have investigated the steady generalized plane Couette flow of viscous incompressible fluid between two porous parallel plates through porous medium with magnetic field. We have studied the velocity, average velocity, shear stress, skin frictions, the volumetric flow, drag coefficients \& stream lines.

Keywords: Steady Couette flow, viscous parallel plates, incompressible fluid,porous medium, \& magnetic field.

Copyright @ 2020: This is an open-access article distributed under the terms of the Creative Commons Attribution license which permits unrestricted use, distribution, and reproduction in any medium for non-commercial use (Non Commercial, or CC-BY-NC) provided the original author and source are credited.

\section{NOMENCLATURE}

$u=$ Velocity component along $\mathrm{x}$-axis

$v=$ Velocity component along $\mathrm{y}-$ axis

$t=$ The time

$\rho=$ The density of fluid

$P=$ The fluid pressure

$k=$ The thermal conductivity

$\mu=$ Coefficient of viscosity

$v=$ Kinematic viscosity

$Q=$ The volumetric flow

\section{INTRODUCTION}

We have investigated steady generalized plane Couette flow of viscous incompressible fluid between two porous parallel plates through porous mediumwith magnetic field. Attempts have been made by several researchers i.e. M. Aydin \& R. T. Fenner [1] investigated boundary element analysis of driven cavity flow for low \& moderate Reynolds number. N. Bahloul \& Boutana \& P. Vasseur [2] investigated double diffusive \& sorbet induced convection in a shallow horizontal porous layer. V. et. al. Barbu [3] investigated exact controllability magneto hydrodynamic equations. D. Barkley \& L. S. Tuckerman [4] investigated stability analysis of perturbed plane Couette flow. D. Barkley \& L. S. Tuckerman [5] investigated turbulent laminar patterns in plane Couette flow. D. Barkley \& L. S. Tuckerman [6] investigated mean flow of turbulentlaminar patterns in plane Couette flow. E. Barragy \& G. F. Carey [7] investigated stream function vorticity driven cavity solutions using $\mathrm{p}$ finite elements. G. K. Batchelor [8] investigated a proposal concerning laminar wakes behind bluff body's at large Reynolds number. G. K. Batchelor [9] investigated on steady laminar flow with closed streamlines at large Reynolds number. C. Baytas \& I. Pop [10] investigated free convection in oblique enclosures filled with a porous medium. R. M. Beam \& R. F. warming [11] investigated an implicit factored scheme for the compressible Navier-Stokes equations. Beant Singh \& Chanpreet Singh [12] investigated analysis of vortex motion in porous media. R. Sulc \& P. Ditl [13] investigated local energy dissipation rate in an agitated vessel a comparison of evaluation methods. R. Šulc, P. Ditl, I. Fořt, D. Jašíkova, M. Kotek, V. Kopecký \& B. Kysela [14] investigated the effect of particle image velocimetry setting parameters on local velocity measurements in an agitated vessel. G. G. Tsypkin \& V. A. Shargatov [15] investigated influence of capillary pressure gradient on connectivity of flow through a porous medium. In this paperwe have investigated the velocity, average velocity, shear stress, skin frictions, the volumetric flow, drag coefficients and stream lines. 


\section{FORMULATION OF THE PROBLEM}

Let us consider two infinite porous plates $\mathrm{AB} \& \mathrm{CD}$ separated by a distance $2 \mathrm{~h}$. The fluid enters in y direction. The velocity component along $\mathrm{x}$-axis is a function of $\mathrm{y}$ only. The motion of incompressible fluid is in two dimension and is steady then

$$
u=u(y), \quad w=0 \quad \& \quad \frac{\partial}{\partial t} \equiv 0
$$

The equation of continuity for incompressible fluid

$\frac{\partial u}{\partial x}+\frac{\partial v}{\partial y}+\frac{\partial w}{\partial z}=0$ put $w=0 \& \frac{\partial u}{\partial x}=0 \Rightarrow \frac{\partial v}{\partial y}=0$

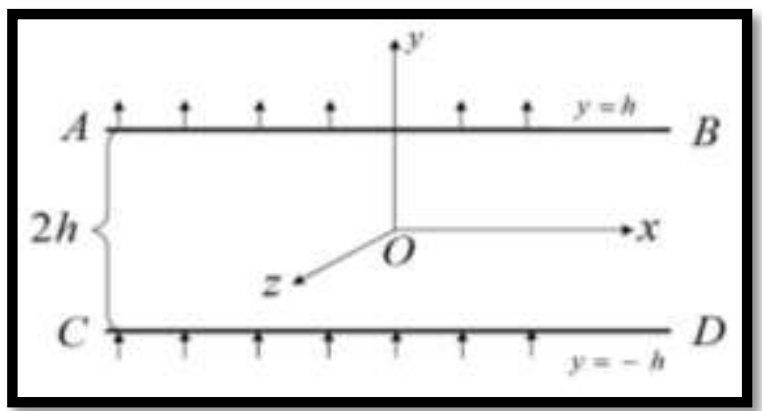

Figure-1

$v$ Is independent of $y$ but motion is along y-axis. So we can say that $v$ is constant velocity i.e. $v=v_{0}$ or the fluid enters in flow region through one plate at the same constant velocity $\mathrm{v}_{0}$.

Also Navier-Stokes equations for incompressible fluid in the absence of body force when flow is steady

$$
v_{0} \frac{d u}{d y}=-\frac{1}{\rho} \frac{\partial p}{\partial x}+v \frac{d^{2} u}{d y^{2}}+\left(\frac{1}{k}+\frac{\sigma B_{0}^{2}}{\mu}\right) v u \ldots \ldots \ldots . .(1) \&-\frac{1}{\rho} \frac{\partial p}{\partial y}=0 \ldots
$$

\section{SOLUTION OF THE PROBLEM}

Equation (2) shows that the pressure does not depend on $y$ hence $p$ is a function of $x$ only \& so equation (1) reduces to

$$
\frac{d p}{d x}=\rho\left(v \frac{d^{2} u}{d y^{2}}-v_{0} \frac{d u}{d y}+\frac{v u}{k}+\frac{\sigma B_{0}^{2} v u}{\mu}\right) \Rightarrow \frac{d^{2} u}{d y^{2}}-\frac{v_{0}}{v} \frac{d u}{d y}+\left(\frac{1}{k}+\frac{\sigma B_{0}^{2}}{\mu}\right) u=-\frac{P}{\rho v} \text { where } \frac{d p}{d x}=-P
$$

A.E. $m^{2}-\frac{v_{0}}{v} m+\left(\frac{1}{k}+\frac{\sigma B_{0}^{2}}{\mu}\right)=0 \Rightarrow m=\frac{\frac{v_{0}}{v} \pm \sqrt{\left(\frac{v_{0}}{v}\right)^{2}-4\left(\frac{1}{k}+\frac{\sigma B_{0}^{2}}{\mu}\right)}}{2}=\frac{v_{0}}{2 v} \pm \sqrt{\left(\frac{v_{0}}{2 v}\right)^{2}-\left(\frac{1}{k}+\frac{\sigma B_{0}^{2}}{\mu}\right)}$

Let $\sqrt{\left(\frac{v_{0}}{2 v}\right)^{2}-\left(\frac{1}{k}+\frac{\sigma B_{0}^{2}}{\mu}\right)}=A \quad, \quad \frac{1}{k}+\frac{\sigma B_{0}^{2}}{\mu}=B \quad \&\left(\frac{v_{0}}{2 v}\right)^{2}>\left(\frac{1}{k}+\frac{\sigma B_{0}^{2}}{\mu}\right)$

$\therefore C . F .=e^{\frac{v_{0}}{2 v} y}\left[C_{1} \operatorname{Cosh} A y+C_{2} \operatorname{Sinh} A y\right] \& \quad P . I .=-\frac{P}{\mu B} \Rightarrow u(y)=e^{\frac{v_{0}}{2 v} y}\left[C_{1} \operatorname{Cosh} A y+C_{2} \operatorname{Sinh} A y\right]-\frac{P}{\mu B}$

Using boundary conditions

$$
u=0 \text { at } y=-h \quad \& \quad u=U \text { at } y=h
$$

$$
e^{-\frac{v_{0}}{2 v} h}\left[C_{1} \operatorname{Cosh} A h-C_{2} \operatorname{Sinh} A h\right]-\frac{P}{\mu B}=0
$$

(3) \& $\quad U=e^{\frac{v_{0}}{2 v} h}\left[C_{1} \operatorname{Cosh} A h+C_{2} \operatorname{Sinh} A h\right]-\frac{P}{\mu B}$.

$\Rightarrow \frac{P}{\mu B} e^{\frac{v_{0}}{2 v} h}=C_{1} \operatorname{Cosh} A h-C_{2} \operatorname{Sinh} A h \&\left(U+\frac{P}{\mu B}\right) e^{-\frac{v_{0}}{2 v} h}=C_{1} \operatorname{Cosh} A h+C_{2} \operatorname{Sinh} A h$

$C_{1}=\frac{1}{2 \operatorname{Cosh} A h}\left[\left(U+\frac{P}{\mu B}\right) e^{-\frac{v_{0}}{2 v} h}+\frac{P}{\mu B} e^{\frac{v_{0}}{2 v} h}\right] \& C_{2}=\frac{1}{2 \operatorname{Sinh} A h}\left[\left(U+\frac{P}{\mu B}\right) e^{-\frac{v_{0}}{2 v} h}-\frac{P}{\mu B} e^{\frac{v_{0}}{2 v} h}\right]$ 


$$
\begin{gathered}
u(y)=\frac{e^{\frac{v_{0}}{2 v} y} \operatorname{Cosh} A y}{2 \operatorname{Cosh} A h}\left\{\left(U+\frac{P}{\mu B}\right) e^{-\frac{v_{0}}{2 v} h}+\frac{P}{\mu B} e^{\frac{v_{0}}{2 v} h}\right\}+\frac{e^{\frac{v_{0}}{2 v} y} \operatorname{Sinh} A y}{2 \operatorname{Sinh} A h}\left\{\left(U+\frac{\rho}{\mu B}\right) e^{-\frac{v_{0}}{2 v} h}-\frac{P}{\mu B} e^{\frac{v_{0}}{2 v} h}\right\}-\frac{P}{\mu B} \\
u(y)=\left(U+\frac{P}{\mu B}\right) \frac{e^{\frac{v_{0}}{2 v}(y-h)} \operatorname{Sinh} A(y+h)}{2 \operatorname{Sinh} A h \operatorname{Cosh} A h}-\frac{P}{\mu B} \frac{e^{\frac{v_{0}}{2 v}(y+h)} \operatorname{Sinh} A(y-h)}{2 \operatorname{Sinh} A h \operatorname{Cosh} A h}-\frac{P}{\mu B} \\
u(y)=\frac{1}{\operatorname{Sinh} 2 A h}\left[\left(U+\frac{P}{\mu B}\right) e^{\frac{v_{0}}{2 v}(y-h)} \operatorname{Sinh} A(y+h)-\frac{P}{\mu B} e^{\frac{v_{0}}{2 v}(y+h)} \operatorname{Sinh} A(y-h)\right]-\frac{P}{\mu B} \ldots \ldots \ldots . . .(5)
\end{gathered}
$$

\section{THE SHEAR STRESS AT ANY POINT}

$$
\sigma_{x y}=\frac{\mu}{\operatorname{Sinh} 2 A h}\left[\left(U+\frac{P}{\mu B}\right) e^{\frac{v_{0}}{2 v}(y-h)}\left\{\frac{v_{0}}{2 v} \operatorname{Sinh} A(y+h)+A \operatorname{Cosh} A(y+h)\right\}-\frac{P}{\mu B} e^{\frac{v_{0}}{2 v}(y+h)}\left\{\frac{v_{0}}{2 v} \operatorname{Sinh} A(y-h)+A \operatorname{Cosh} A(y-h)\right\} \ldots \ldots\right.
$$

\section{THE SKIN FRICTIONS AT LOWER AND UPPER PLATE}

$$
\begin{aligned}
& \left(\sigma_{x y}\right)_{y=-h}=\frac{\mu}{\operatorname{Sinh} 2 A h}\left[A\left(U+\frac{P}{\mu B}\right) e^{-\frac{v_{0}}{v} h}+\frac{P}{\mu B}\left\{\frac{v_{0}}{2 v} \operatorname{Sinh} 2 A h-A \operatorname{Cosh} 2 A h\right\}\right] \\
& \left(\sigma_{x y}\right)_{y=h}=\frac{\mu}{\operatorname{Sinh} 2 A h}\left[\left(U+\frac{P}{\mu B}\right)\left(\frac{v_{0}}{2 v} \operatorname{Sinh} 2 A h+A \operatorname{Cosh} 2 A h\right)-\frac{P A}{\mu B} e^{\frac{v_{0}}{v} h}\right] \ldots
\end{aligned}
$$

\section{THE AVERAGE VELOCITY DISTRIBUTION IN GENERALIZED PLANE COUETTE FLOW}

$$
\begin{aligned}
& (u)_{a v}=\frac{1}{2 h} \int_{-h}^{h} u(y) d y=\frac{1}{2 h} \int_{-h}^{h}\left[\frac{1}{\operatorname{Sinh} 2 A h}\left\{\left(U+\frac{P}{\mu B}\right) e^{\frac{v_{0}}{2 v}(y-h)} \operatorname{Sinh} A(y+h)-\frac{P}{\mu B} e^{\frac{v_{0}}{2 v}(y+h)} \operatorname{Sinh} A(y-h)\right\}-\frac{P}{\mu B}\right] d y \\
& =\frac{\left(U+\frac{P}{\mu B}\right)}{4 h \operatorname{Sinh} 2 A h} \int_{-h}^{h}\left\{e^{\frac{v_{0}}{2 v}(y-h)+A(y+h)}-e^{\frac{v_{0}}{2 v}(y-h)-A(y+h)}\right\} d y-\frac{P}{4 h \mu B \operatorname{Sinh} 2 A h} \int_{-h}^{h}\left\{e^{\frac{v_{0}}{2 v}(y+h)+A(y-h)}-e^{\frac{v_{0}}{2 v}(y+h)-A(y-h)}\right\} d y-\frac{P}{\mu B} \\
& =\frac{\left(U+\frac{P}{\mu B}\right)}{4 h \operatorname{Sinh} 2 A h}\left\{\frac{e^{\frac{v_{0}}{2 v}(y-h)+A(y+h)}}{\left(\frac{v_{0}}{2 v}+A\right)}-\frac{e^{\frac{v_{0}}{2 v}(y-h)-A(y+h)}}{\left(\frac{v_{0}}{2 v}-A\right)}\right\}_{-h}^{h}-\frac{P}{4 \mu h B \operatorname{Sinh} 2 A h}\left\{\frac{e^{\frac{v_{0}}{2 v}(y+h)+A(y-h)}}{\left(\frac{v_{0}}{2 v}+A\right)}-\frac{e^{\frac{v_{0}}{2 v}(y+h)-A(y-h)}}{\left(\frac{v_{0}}{2 v}-A\right)}\right\}_{-h}^{h}-\frac{P}{\mu B} \\
& =\frac{\left(U+\frac{P}{\mu B}\right)}{4 h \operatorname{Sinh} 2 A h}\left[\frac{\left(e^{2 A h}-e^{-\frac{v_{0}}{v} h}\right)}{\left(\frac{v_{0}}{2 v}+A\right)}-\frac{\left(e^{-2 A h}-e^{-\frac{v_{0}}{v} h}\right)}{\left(\frac{v_{0}}{2 v}-A\right)}\right]-\frac{P}{4 \mu h B \operatorname{Sinh} 2 A h}\left[\frac{\left(e^{\frac{v_{0}}{v} h}-e^{-2 A h}\right)}{\left(\frac{v_{0}}{2 v}+A\right)}-\frac{\left(e^{\frac{v_{0}}{v} h}-e^{2 A h}\right)}{\left(\frac{v_{0}}{2 v}-A\right)}\right]-\frac{P}{\mu B} \\
& =\frac{\left(U+\frac{P}{\mu B}\right)}{4 h \operatorname{Sinh} 2 A h}\left[\frac{\left(\frac{v_{0}}{2 v}-A\right)\left(e^{2 A h}-e^{-\frac{v_{0}}{v} h}\right)-\left(\frac{v_{0}}{2 v}+A\right)\left(e^{-2 A h}-e^{-\frac{v_{0}}{v} h}\right)}{\left\{\left(\frac{v_{0}}{2 v}\right)^{2}-A^{2}\right\}}\right]-\frac{P}{4 \mu h B \operatorname{Sinh} 2 A h}\left[\frac{\left(\frac{v_{0}}{2 v}-A\right)\left(e^{\frac{v_{0}}{v} h}-e^{-2 A h}\right)-\left(\frac{v_{0}}{2 v}+A\right)\left(e^{\frac{v_{0}}{v} h}-e^{2 A h}\right)}{\left\{\left(\frac{v_{0}}{2 v}\right)^{2}-A^{2}\right\}}\right]-\frac{P}{\mu B} \\
& =\frac{\left(U+\frac{P}{\mu B}\right)}{4 B h \operatorname{Sinh} 2 A h}\left\{\frac{v_{0}}{2 v}\left(e^{2 A h}-e^{-\frac{v_{0}}{v} h}-e^{-2 A h}+e^{-\frac{v_{0}}{v} h}\right)-A\left(e^{2 A h}-e^{-\frac{v_{0}}{v} h}+e^{-2 A h}-e^{-\frac{v_{0}}{v} h}\right)\right\}
\end{aligned}
$$




$$
-\frac{P}{4 \mu h B^{2} \operatorname{Sinh} 2 A h}\left\{\frac{v_{0}}{2 v}\left(e^{\frac{v_{0}}{v} h}-e^{-2 A h}-e^{\frac{v_{0}}{v} h}+e^{2 A h}\right)-A\left(e^{\frac{v_{0}}{v} h}-e^{-2 A h}+e^{\frac{v_{0}}{v} h}-e^{2 A h}\right)\right\}-\frac{P}{\mu B}
$$

Since $\sqrt{\left(\frac{v_{0}}{2 v}\right)^{2}-\left(\frac{1}{k}+\frac{\sigma B_{0}^{2}}{\mu}\right)}=A \quad, \quad\left(\frac{1}{k}+\frac{\sigma B_{0}^{2}}{\mu}\right)=B \Rightarrow\left(\frac{v_{0}}{2 v}\right)^{2}-A^{2}=B$

$=\frac{\left(U+\frac{P}{\mu B}\right)}{2 B h \operatorname{Sinh} 2 A h}\left(\frac{v_{0}}{2 v} \operatorname{Sinh} 2 A h-A \operatorname{Cosh} 2 A h+A e^{-\frac{v_{0}}{v} h}\right)-\frac{P}{2 \mu h B^{2} \operatorname{Sinh} 2 A h}\left(\frac{v_{0}}{2 v} \operatorname{Sinh} 2 A h+A \operatorname{Cosh} 2 A h-A e^{\frac{v_{0}}{v} h}\right)-\frac{P}{\mu B}$

$$
u_{a v}=\frac{U}{2 h B \operatorname{Sinh} 2 A h}\left(\frac{v_{0}}{2 v} \operatorname{Sinh} 2 A h-A \operatorname{Cosh} 2 A h+A e^{-\frac{v_{0}}{v} h}\right)-\frac{P A}{\mu h B^{2} \operatorname{Sinh} 2 A h}\left\{\operatorname{Cosh} 2 A h-\operatorname{Cosh}\left(\frac{v_{0}}{v} h\right)\right\}-\frac{P}{\mu B} \ldots . .
$$

THE VOLUMETRIC FLOW: $Q=2 h(u)_{a v}$

$$
Q=\frac{U}{B \operatorname{Sinh} 2 A h}\left(\frac{v_{0}}{2 v} \operatorname{Sinh} 2 A h-A \operatorname{Cosh} 2 A h+A e^{-\frac{v_{0}}{v} h}\right)-\frac{2 P A}{\mu B^{2} \operatorname{Sinh} 2 A h}\left\{\operatorname{Cosh} 2 A h-\operatorname{Cosh}\left(\frac{v_{0}}{v} h\right)\right\}-\frac{2 h P}{\mu B} .
$$

THE DRAG COEFFICIENTS: $\left(C_{f}\right)_{y=h}=\frac{\left(\sigma_{x y}\right)_{y=h}}{\frac{1}{2} \rho u_{a v}^{2}} \&\left(C_{f}^{\prime}\right)_{y=-h}=\frac{\left(\sigma_{x y}\right)_{y=-h}}{\frac{1}{2} \rho u_{a v}^{2}}$

$$
\begin{gathered}
\left(C_{f}\right)_{y=h}=\frac{8 \mu^{3} h^{2} B^{4} \operatorname{Sinh} 2 A h\left\{\left(U+\frac{P}{\mu B}\right)\left(\frac{v_{0}}{2 v} \operatorname{Sinh} 2 A h+A \operatorname{Cosh} 2 A h\right)-\frac{P A}{\mu B} e^{\frac{v_{0}}{v} h}\right\}}{\rho\left[\mu U B\left(\frac{v_{0}}{2 v} \operatorname{Sinh} 2 A h-A \operatorname{Cosh} 2 A h+A e^{-\frac{v_{0}}{v} h}\right)-2 A P\left\{\operatorname{Cosh} 2 A h-\operatorname{Cosh}\left(\frac{v_{0}}{v} h\right)\right\}-2 P B h \operatorname{Sinh} 2 A h\right]^{2}} . \\
\left(C_{f}^{\prime}\right)_{y=-h}=\frac{8 \mu^{3} h^{2} B^{4} \operatorname{Sinh} 2 A h\left\{A\left(U+\frac{P}{\mu B}\right) e^{-\frac{v_{0}}{v} h}+\frac{P}{\mu B}\left(\frac{v_{0}}{2 v} \operatorname{Sinh} 2 A h-A \operatorname{Cosh} 2 A h\right)\right\}}{\rho\left[\mu U B\left(\frac{v_{0}}{2 v} \operatorname{Sinh} 2 A h-A \operatorname{Cosh} 2 A h+A e^{-\frac{v_{0}}{v} h}\right)-2 A P\left\{\operatorname{Cosh} 2 A h-\operatorname{Cosh}\left(\frac{v_{0}}{v} h\right)\right\}-2 P B h \operatorname{Sinh} 2 A h\right]^{2}} \ldots
\end{gathered}
$$

THE STREAM LINE IN THE PLANE GENERALIZED COUETTE FLOW:

$$
\Rightarrow \frac{\frac{d x}{u}=\frac{d y}{v}=\frac{d z}{w} \quad \text { where } \bar{q}=u \hat{i}+v \hat{j}+w \hat{k}}{\left[\frac{1}{\operatorname{Sinh} 2 A h}\left\{\left(U+\frac{P}{\mu B}\right) e^{\frac{v_{0}}{2 v}(y-h)} \operatorname{Sinh} A(y+h)-\frac{P}{\mu B} e^{\frac{v_{0}}{2 v}(y+h)} \operatorname{Sinh} A(y-h)\right\}-\frac{P}{\mu B}\right]}=\frac{d y}{v_{0}}=\frac{d z}{0}
$$

\section{Taking first two equations}

$v_{0} \int d x=\int\left[\frac{1}{\operatorname{Sinh} 2 A h}\left\{\left(U+\frac{P}{\mu B}\right) e^{\frac{v_{0}}{2 v}(y-h)} \operatorname{Sinh} A(y+h)-\frac{P}{\mu B} e^{\frac{v_{0}}{2 v}(y+h)} \operatorname{Sinh} A(y-h)\right\}-\frac{P}{\mu B}\right] d y+C_{1}$ 


$$
\begin{aligned}
& \Rightarrow v_{0} x-\frac{\left(U+\frac{P}{\mu B}\right)}{\operatorname{Sinh} 2 A h} \int e^{\frac{v_{0}}{2 v}(y-h)}\left\{\frac{e^{A(y+h)}-e^{-A(y+h)}}{2}\right\} d y+\frac{P}{\mu B \operatorname{Sinh} 2 A h} \int e^{\frac{v_{0}}{2 v}(y+h)}\left\{\frac{e^{A(y-h)}-e^{-A(y-h)}}{2}\right\} d y+\frac{P}{\mu B} y=C_{1} \\
& \Rightarrow v_{0} x-\frac{\left(U+\frac{P}{\mu B}\right)}{2 \operatorname{Sinh} 2 A h} \int\left(e^{\frac{v_{0}}{2 v}(y-h)+A(y+h)}-e^{\frac{v_{0}}{2 v}(y-h)-A(y+h)}\right) d y+\frac{P}{2 \mu B \operatorname{Sinh} 2 A h} \int\left(e^{\frac{v_{0}}{2 v}(y+h)+A(y-h)}-e^{\frac{v_{0}}{2 v}(y+h)-A(y-h)}\right) d y+\frac{P}{\mu B} y=C_{1} \\
& \Rightarrow v_{0} x-\frac{\left(U+\frac{P}{\mu B}\right)}{2 \operatorname{Sinh} 2 A h}\left(\frac{e^{\frac{v_{0}}{2 v}(y-h)+A(y+h)}}{\left(\frac{v_{0}}{2 v}+A\right)}-\frac{e^{\frac{v_{0}}{2 v}(y-h)-A(y+h)}}{\left(\frac{v_{0}}{2 v}-A\right)}\right)+\frac{P}{2 \mu B \operatorname{Sinh} 2 A h}\left(\frac{e^{\frac{v_{0}}{2 v}(y+h)+A(y-h)}}{\left(\frac{v_{0}}{2 v}+A\right)}-\frac{e^{\frac{v_{0}}{2 v}(y+h)-A(y-h)}}{\left(\frac{v_{0}}{2 v}-A\right)}\right)+\frac{P}{\mu B} y=C_{1} \\
& \Rightarrow v_{0} x-\frac{\left(U+\frac{P}{\mu B}\right) e^{\frac{v_{0}}{2 v}(y-h)}}{2 B \operatorname{Sinh} 2 A h}\left\{\left(\frac{v_{0}}{2 v}-A\right) e^{A(y+h)}-\left(\frac{v_{0}}{2 v}+A\right) e^{-A(y+h)}\right\}+\frac{P e^{\frac{v_{0}}{2 v}(y+h)}}{2 \mu B^{2} \operatorname{Sinh} 2 A h}\left\{\left(\frac{v_{0}}{2 v}-A\right) e^{A(y-h)}-\left(\frac{v_{0}}{2 v}+A\right) e^{-A(y-h)}\right\}+\frac{P}{\mu B} y=C_{1} \\
& \Rightarrow v_{0} x-\frac{\left(U+\frac{P}{\mu B}\right) e^{\frac{v_{0}}{2 v}(y-h)}}{B \operatorname{Sinh} 2 A h}\left(\frac{v_{0}}{2 v} \operatorname{Sinh} A(y+h)-A \operatorname{Cosh} A(y+h)\right)+\frac{P e^{\frac{v_{0}}{2 v}(y+h)}}{\mu B^{2} \operatorname{Sinh} 2 A h}\left(\frac{v_{0}}{2 v} \operatorname{Sinh} A(y-h)-A \operatorname{Cosh} A(y-h)\right)+\frac{P}{\mu B} y=C_{1} \cdots
\end{aligned}
$$$$
\text { \& Second stream line is given by } z=C_{2}
$$

\& Second stream line is given by $z=C_{2}$

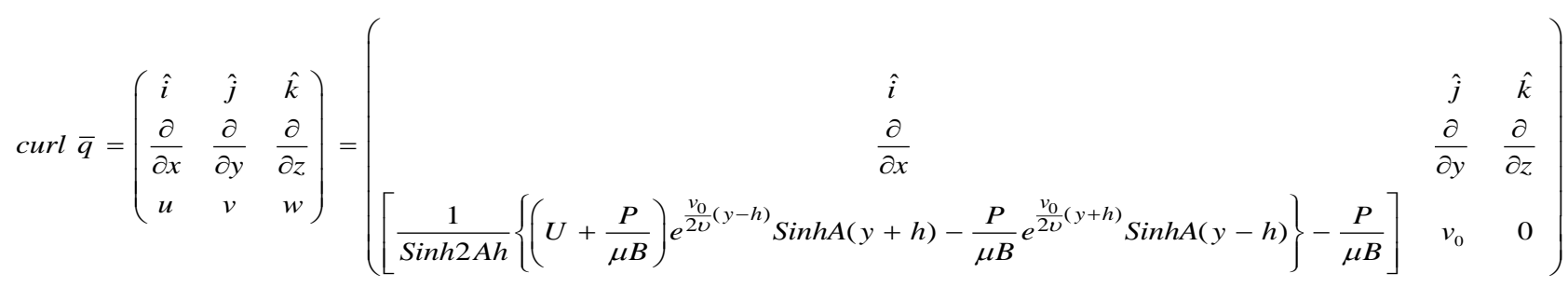

$\operatorname{curl} \bar{q}=-\frac{1}{\operatorname{Sinh} 2 A h}\left[\left(U+\frac{P}{\mu B}\right) e^{\frac{v_{0}}{2 v}(y-h)}\left\{\frac{v_{0}}{2 v} \operatorname{Sinh} A(y+h)+A \operatorname{Cosh} A(y+h)\right\}-\frac{P}{\mu B} e^{\frac{v_{0}}{2 v}(y+h)}\left\{\frac{v_{0}}{2 v} \operatorname{Sinh} A(y-h)+A \operatorname{Cosh} A(y-h)\right\}\right] \hat{k} \neq \overline{0}$ $\Rightarrow$ motion of the fluid is rotational.

Table for velocity: $P=9, U=6, \mu=.5, h=.5, \frac{v_{0}}{2 v}=6 \& \frac{1}{k}=\frac{\sigma B_{0}^{2}}{\mu}=16, \sqrt{\left(\frac{v_{0}}{2 v}\right)^{2}-\left(\frac{1}{k}+\frac{\sigma B_{0}^{2}}{\mu}\right)}=2$

Table-1: (for velocity)

\begin{tabular}{|c|c|c|c|c|c|c|c|c|}
\hline & $\mathrm{y}$ & $\mathrm{0}$ & .1 & .2 & .3 & .4 & .5 & .6 \\
\hline$\frac{1}{k}=16$ & $\mathrm{u}(\mathrm{y})$ & 1.3 & .645 & 1.301 & 1.882 & 1.835 & 6 & -7.818 \\
\hline$\frac{\sigma B_{0}^{2}}{\mu}=16$ & $\mathrm{u}(\mathrm{y})$ & 1.3 & .645 & 1.301 & 1.882 & 1.835 & 6 & -7.818 \\
\hline$\frac{1}{k}+\frac{\sigma B_{0}^{2}}{\mu}=32$ & $\mathrm{u}(\mathrm{y})$ & 3.246 & 4.726 & 6.592 & 8.473 & 9.276 & 6 & -8.82 \\
\hline
\end{tabular}




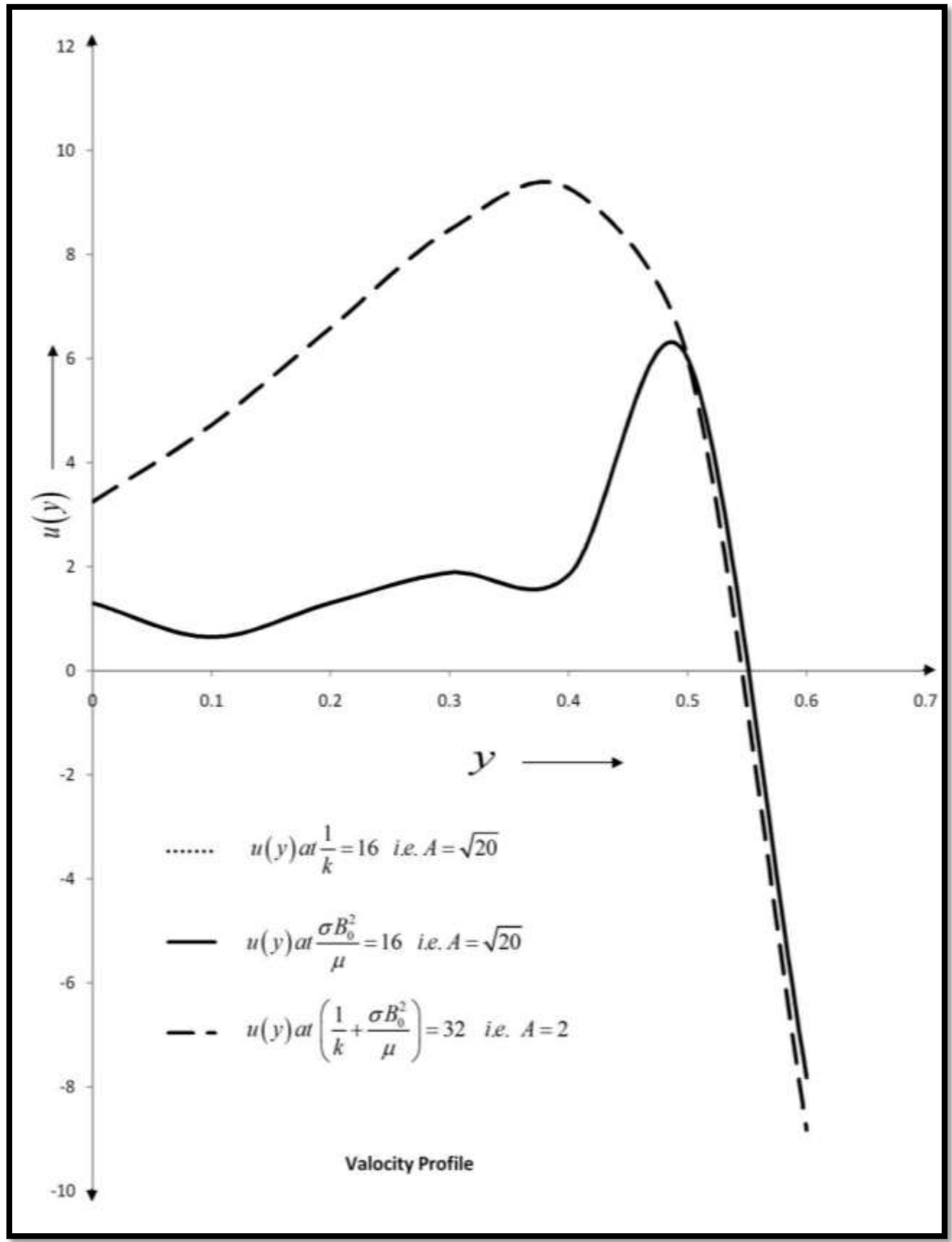

Graph of table-1 
Table for skin friction: $P=9, U=6, \mu=h=.5, \frac{v_{0}}{2 v}=6 \& \frac{1}{k}=\frac{\sigma B_{0}^{2}}{\mu}=16, \sqrt{\left(\frac{v_{0}}{2 v}\right)^{2}-\left(\frac{1}{k}+\frac{\sigma B_{0}^{2}}{\mu}\right)}=2$

Table-2: (for skin friction)

\begin{tabular}{|c|c|c|c|c|c|c|c|c|}
\hline & $\mathrm{y}$ & $\mathbf{0}$ & .1 & .2 & .3 & .4 & .5 & .6 \\
\hline$\frac{1}{k}=16$ & $\sigma_{x y}$ & $\mathbf{1 . 8 9 9}$ & $-\mathbf{4 . 6 8 5}$ & $-\mathbf{1 0 . 3 0 4}$ & -22.184 & $-\mathbf{4 7 . 0 3 8}$ & 14.123 & -205.03 \\
\hline$\frac{\sigma B_{0}^{2}}{\mu}=16$ & $\sigma_{x y}$ & 1.899 & -4.685 & -10.304 & -22.184 & -47.038 & 14.123 & -205.03 \\
\hline$\frac{1}{k}+\frac{\sigma B_{0}^{2}}{\mu}=32$ & $\sigma_{x y}$ & $\mathbf{6 . 6 3 2}$ & $\mathbf{8 . 5 7 2}$ & $\mathbf{9 . 8 4 5}$ & $\mathbf{8 . 1 3 8}$ & -2.436 & $-\mathbf{3 6 . 0 7}$ & -126.012 \\
\hline
\end{tabular}

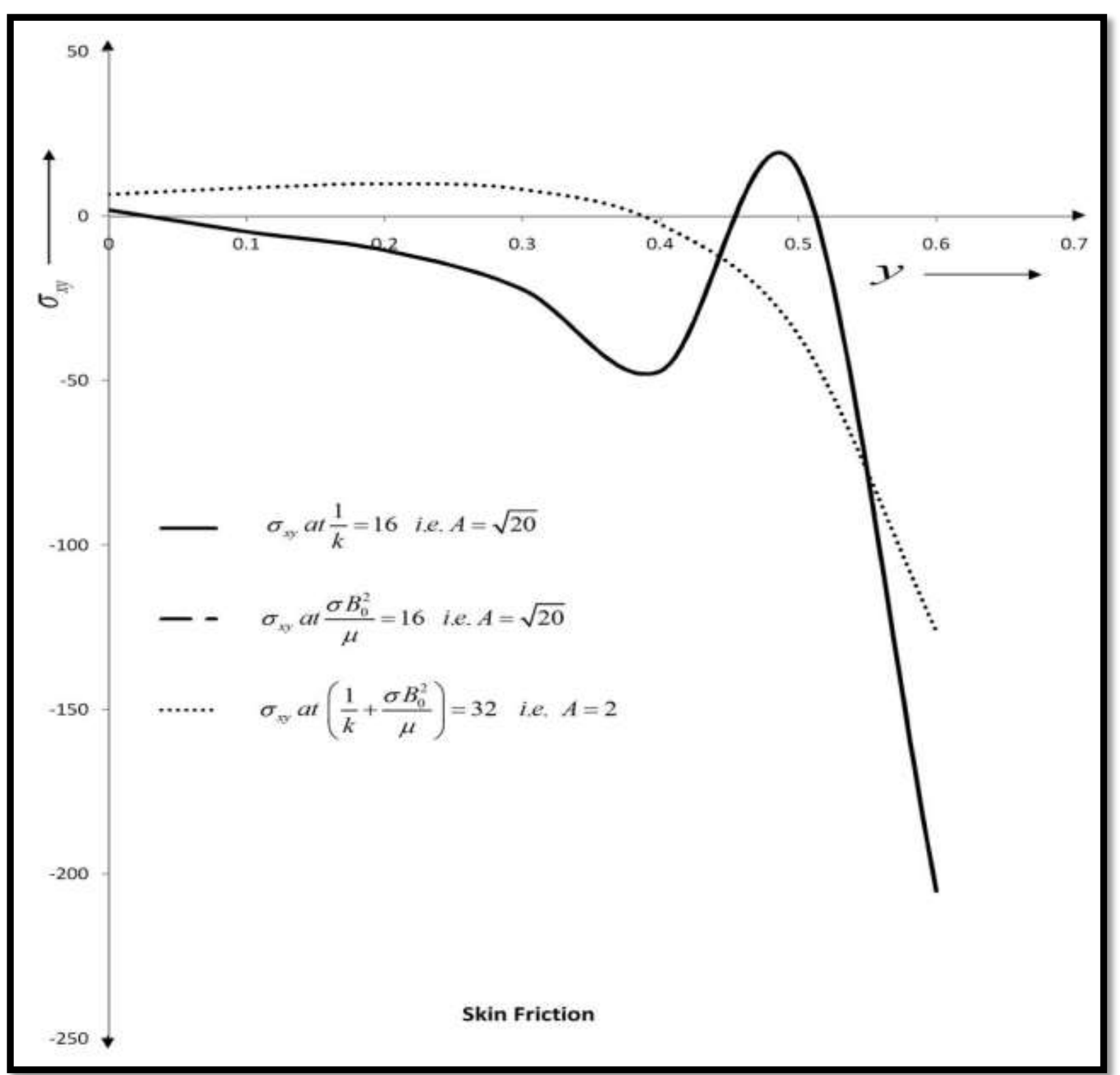

Graph of table-2 
Table for velocity: $P=9, U=6, \mu=h=.5, \frac{v_{0}}{2 v}=6, \sqrt{\left(\frac{v_{0}}{2 v}\right)^{2}-\left(\frac{1}{k}+\frac{\sigma B_{0}^{2}}{\mu}\right)}=2$ when $\frac{1}{k}<\frac{\sigma B_{0}^{2}}{\mu}$

Table-3: (for velocity)

\begin{tabular}{|c|c|c|c|c|c|c|c|c|}
\hline & $\mathrm{y}$ & $\mathbf{0}$ & .1 & .2 & .3 & .4 & .5 & .6 \\
\hline$\frac{1}{k}=11$ & $\mathrm{u}(\mathrm{y})$ & 1.074 & 1.384 & 1.776 & 2.359 & 3.45 & 6 & 12.858 \\
\hline$\frac{\sigma B_{0}^{2}}{\mu}=21$ & $\mathrm{u}(\mathrm{y})$ & .617 & 1.283 & 2.087 & 2.818 & 2.829 & 6 & -8.339 \\
\hline$\frac{1}{k}+\frac{\sigma B_{0}^{2}}{\mu}=32$ & $\mathrm{u}(\mathrm{y})$ & 3.246 & 4.726 & 6.592 & 8.473 & 9.276 & 6 & -8.82 \\
\hline
\end{tabular}

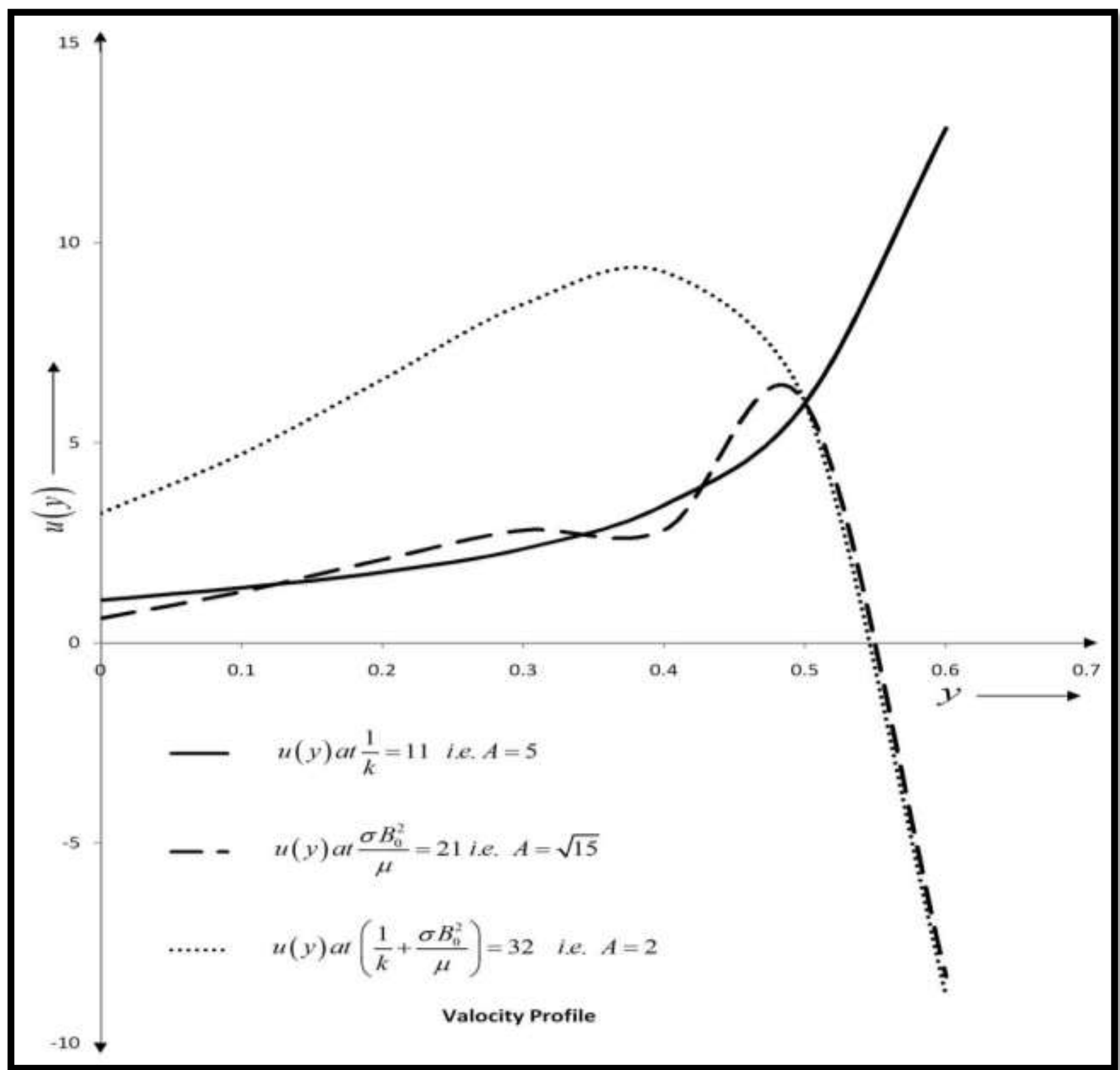

Graph of table-3 
Table for skin friction: $P=9, U=6, \mu=h=.5, \frac{v_{0}}{2 v}=6, \sqrt{\left(\frac{v_{0}}{2 v}\right)^{2}-\left(\frac{1}{k}+\frac{\sigma B_{0}^{2}}{\mu}\right)}=2$ when $\frac{1}{k}<\frac{\sigma B_{0}^{2}}{\mu}$

Table-4: (for skin friction)

\begin{tabular}{|c|c|c|c|c|c|c|c|c|}
\hline & $\mathrm{y}$ & $\mathbf{0}$ & .1 & .2 & .3 & .4 & .5 & .6 \\
\hline$\frac{1}{k}=11$ & $\sigma_{x y}$ & 1.421 & 1.706 & 2.294 & $\mathbf{3 . 7 6 4}$ & $\mathbf{7 . 8 5}$ & 19.76 & $\mathbf{5 5 . 1 4}$ \\
\hline$\frac{\sigma B_{0}^{2}}{\mu}=21$ & $\sigma_{x y}$ & -1.515 & -3.923 & -9.372 & -21.269 & -46.78 & 5.998 & -213.56 \\
\hline$\frac{1}{k}+\frac{\sigma B_{0}^{2}}{\mu}=32$ & $\sigma_{x y}$ & $\mathbf{6 . 6 3 2}$ & $\mathbf{8 . 5 7 2}$ & $\mathbf{9 . 8 4 5}$ & $\mathbf{8 . 1 3 8}$ & $-\mathbf{2 . 4 3 6}$ & $\mathbf{- 3 6 . 0 7}$ & $-\mathbf{1 2 6 . 0 1 2}$ \\
\hline
\end{tabular}

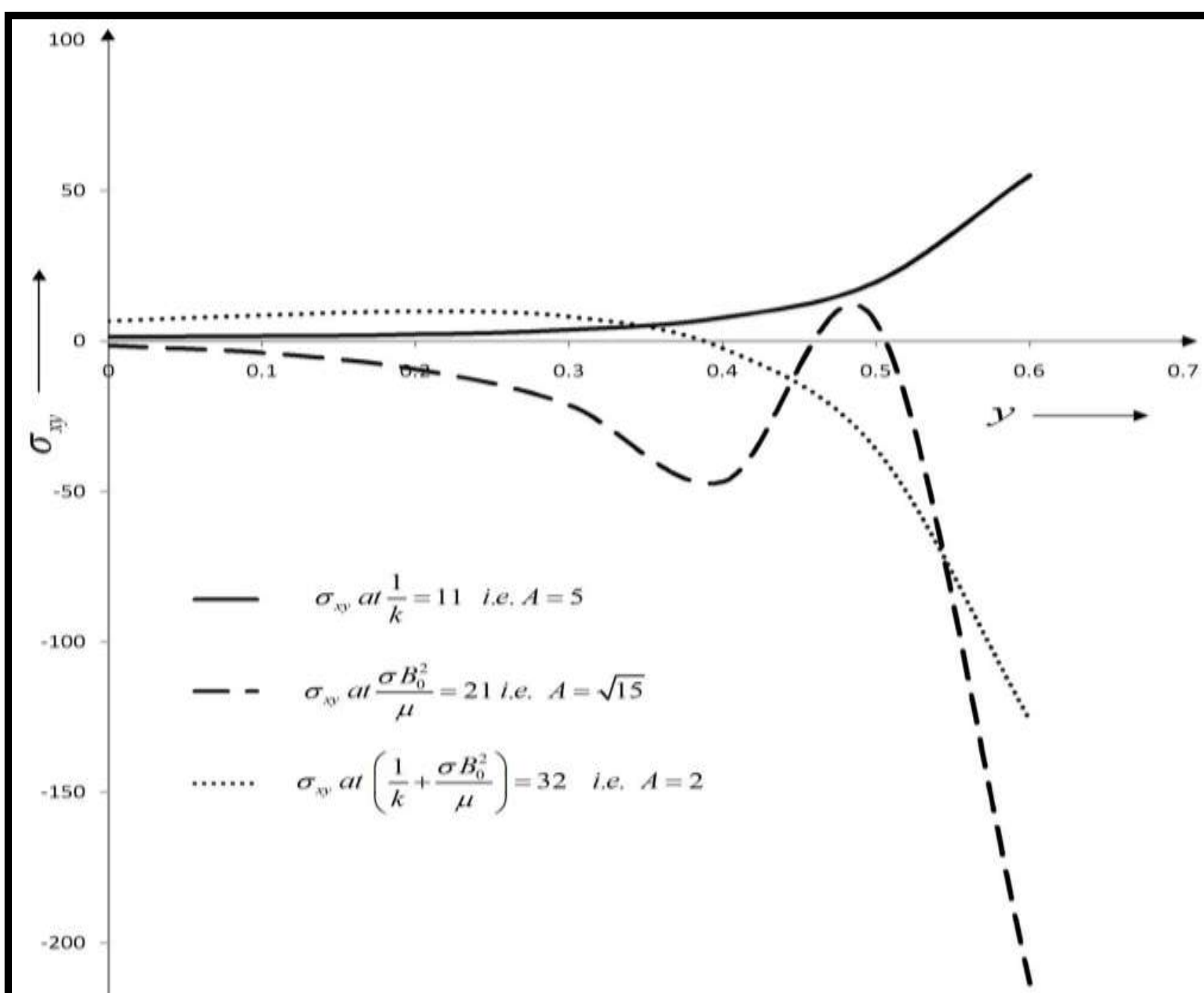


Table for velocity: $P=9, U=6, \mu=h=.5, \frac{v_{0}}{2 v}=6, \sqrt{\left(\frac{v_{0}}{2 v}\right)^{2}-\left(\frac{1}{k}+\frac{\sigma B_{0}^{2}}{\mu}\right)}=2$ when $\frac{1}{k}>\frac{\sigma B_{0}^{2}}{\mu}$

Table-5: (for velocity)

\begin{tabular}{|c|c|c|c|c|c|c|c|c|}
\hline & $\mathrm{y}$ & $\mathrm{0}$ & .1 & .2 & .3 & .4 & .5 & .6 \\
\hline$\frac{1}{k}=21$ & $\mathrm{u}(\mathrm{y})$ & .617 & 1.283 & 2.087 & 2.818 & 2.829 & 6 & -8.339 \\
\hline$\frac{\sigma B_{0}^{2}}{\mu}=11$ & $\mathrm{u}(\mathrm{y})$ & 1.074 & 1.384 & 1.776 & 2.359 & 3.45 & 6 & 12.858 \\
\hline$\frac{1}{k}+\frac{\sigma B_{0}^{2}}{\mu}=32$ & $\mathrm{u}(\mathrm{y})$ & 3.246 & 4.726 & 6.592 & 8.473 & 9.276 & 6 & -8.82 \\
\hline
\end{tabular}

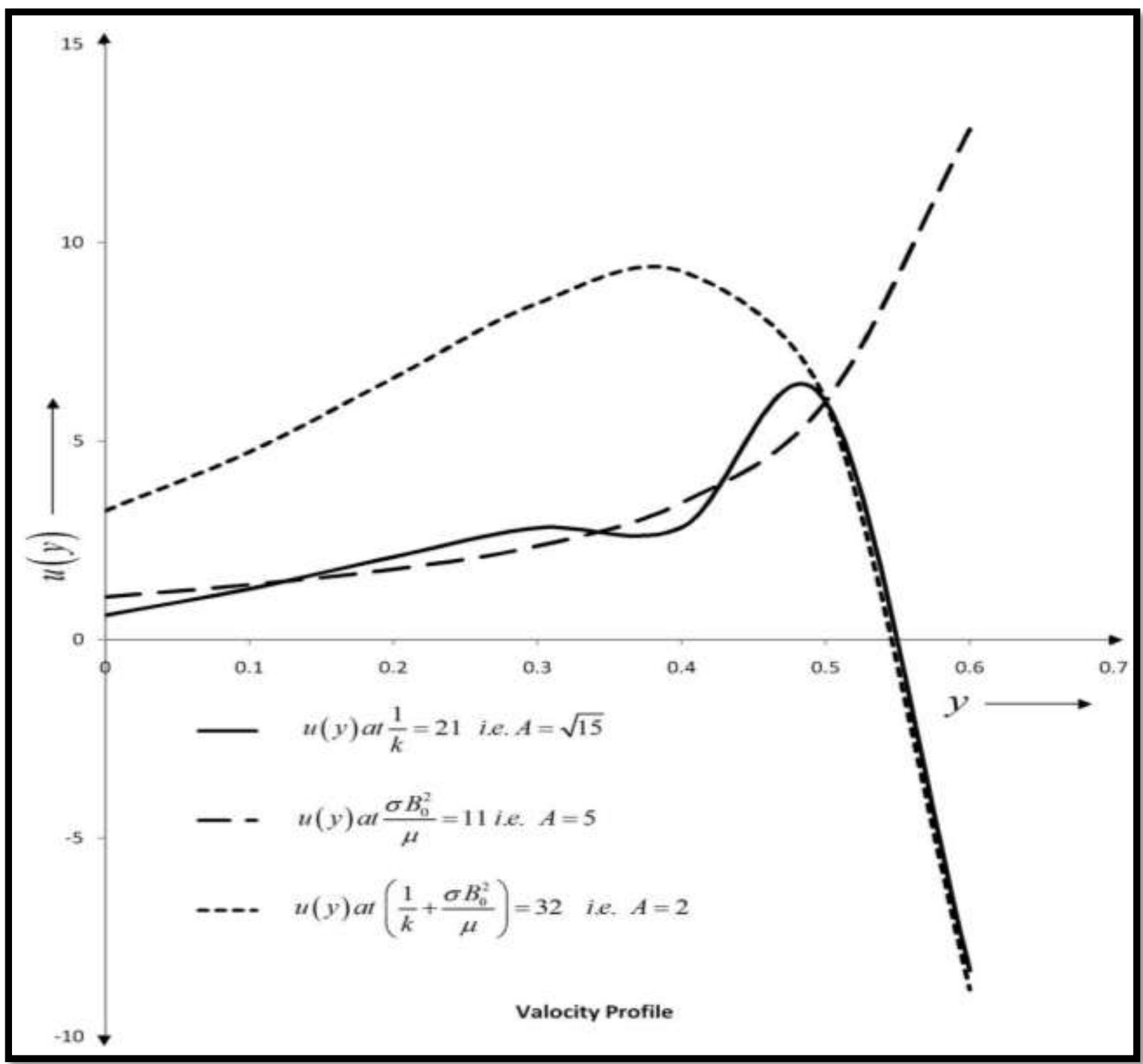

Graph of table-5 
Table for Skin friction: $P=9, U=6, \mu=h=.5, \frac{v_{0}}{2 v}=6, \sqrt{\left(\frac{v_{0}}{2 v}\right)^{2}-\left(\frac{1}{k}+\frac{\sigma B_{0}^{2}}{\mu}\right)}=2$ when $\frac{1}{k}>\frac{\sigma B_{0}^{2}}{\mu}$

Table-6: (for skin friction)

\begin{tabular}{|c|c|c|c|c|c|c|c|c|}
\hline & $\mathrm{y}$ & $\mathbf{0}$ & .1 & .2 & .3 & .4 & .5 & .6 \\
\hline$\frac{1}{k}=21$ & $\sigma_{x y}$ & -1.515 & $-\mathbf{3 . 9 2 3}$ & $-\mathbf{9 . 3 7 2}$ & $-\mathbf{2 1 . 2 6 9}$ & -46.78 & $\mathbf{5 . 9 9 8}$ & -213.56 \\
\hline$\frac{\sigma B_{0}^{2}}{\mu}=11$ & $\sigma_{x y}$ & 1.421 & 1.706 & 2.294 & 3.764 & 7.85 & 19.76 & 55.14 \\
\hline$\frac{1}{k}+\frac{\sigma B_{0}^{2}}{\mu}=32$ & $\sigma_{x y}$ & $\mathbf{6 . 6 3 2}$ & $\mathbf{8 . 5 7 2}$ & $\mathbf{9 . 8 4 5}$ & $\mathbf{8 . 1 3 8}$ & $-\mathbf{2 . 4 3 6}$ & $\mathbf{- 3 6 . 0 7}$ & $-\mathbf{1 2 6 . 0 1 2}$ \\
\hline
\end{tabular}

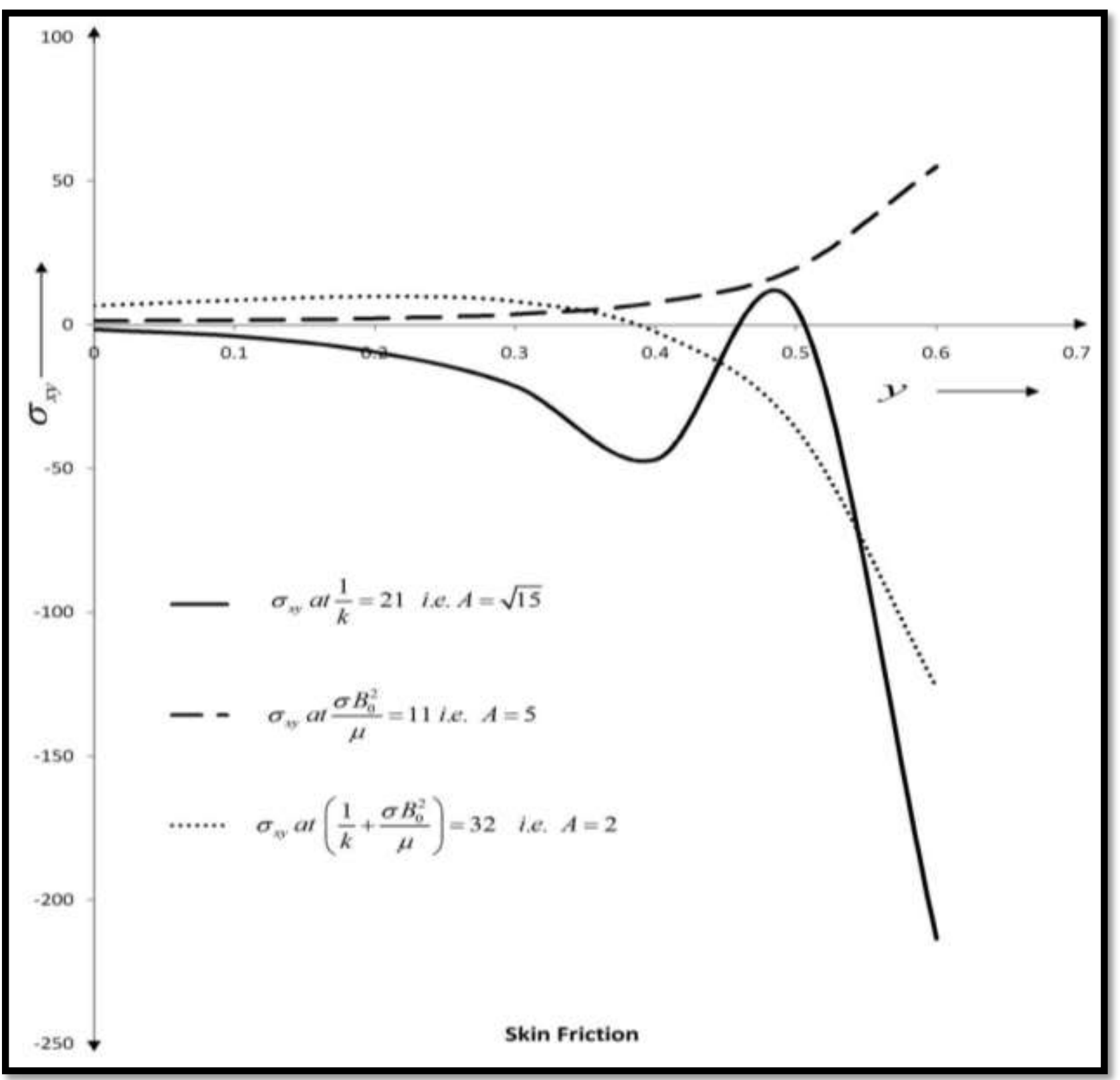

Graph of table-6 


\section{CONCLUSION AND DISCUSSION}

In this paper, we have investigated the velocity by the graph of table- 1 of equation (5). The velocity in porous medium \& magnetic field at $\frac{1}{k}=\frac{\sigma B_{0}^{2}}{\mu}=16$ is less than the corresponding value of velocity in porous with magnetic field at $\frac{1}{k}+\frac{\sigma B_{0}^{2}}{\mu}=32$ in the interval $0 \leq y \leq 0.4 \&$ equal $\{u(y)=6\}$ in all medium at $y=0.5$. The value of velocity in porous medium and magnetic field at $\frac{1}{k}=\frac{\sigma B_{0}^{2}}{\mu}=16$ is negatively less than the correspondingly negative value of velocity in porous medium with magnetic field at $\frac{1}{k}+\frac{\sigma B_{0}^{2}}{\mu}=32$ at $y=0.6$.

Again by the graph of table-3 of equation (5), the value of the velocity in porous medium at $\frac{1}{k}=11$ increases in the interval $0 \leq y \leq 0.6$, velocity in magnetic field at $\frac{\sigma B_{0}^{2}}{\mu}=21$ increases in the interval $0 \leq y \leq 0.5 \&$ the velocity in porous medium with magnetic field at $\frac{1}{k}+\frac{\sigma B_{0}^{2}}{\mu}=32$ increases in the interval $0 \leq y \leq 0.4$. The velocity is equal $\{u(y)=6\}$ in all medium at $y=0.5 \&$ velocity in porous medium at $\frac{1}{k}=11$ is positive while the velocity in magnetic field at $\frac{\sigma B_{0}^{2}}{\mu}=21 \&$ porous medium with magnetic field at $\frac{1}{k}+\frac{\sigma B_{0}^{2}}{\mu}=32$ is negative i.e. flow is in opposite direction at $y=0.6$.

Again by the graph of table-5 of equation (5), the value of the velocity in magnetic field at $\frac{\sigma B_{0}^{2}}{\mu}=11$ increases in the interval $0 \leq y \leq 0.6$, velocity in porous medium at $\frac{1}{k}=21$ increases in the interval $0 \leq y \leq 0.5 \&$ the velocity in porous medium with magnetic field at $\frac{1}{k}+\frac{\sigma B_{0}^{2}}{\mu}=32$ increases in the interval $0 \leq y \leq 0.4$. The velocity is equal $\{u(y)=6\}$ in all medium at $y=0.5 \&$ velocity in magnetic field at $\frac{\sigma B_{0}^{2}}{\mu}=11$ is positive while the velocity in porous medium at $\frac{1}{k}=21 \&$ porous medium with magnetic field at $\frac{1}{k}+\frac{\sigma B_{0}^{2}}{\mu}=32$ is negative i.e. flow is in opposite direction at $y=0.6$.

Again we have investigated the skin friction by the graph of table-2, of equation (6). The skin friction in porous medium \& magnetic field at $\frac{1}{k}=\frac{\sigma B_{0}^{2}}{\mu}=16$ is positive at $y=0 \& .5$ while the skin friction in porous medium \& magnetic field at $\frac{1}{k}=\frac{\sigma B_{0}^{2}}{\mu}=16$ is negative at other values of $\mathrm{y}$. Skin friction in porous medium with magnetic field at $\frac{1}{k}+\frac{\sigma B_{0}^{2}}{\mu}=32$ is positive in the interval $0 \leq y \leq 0.3$ while the skin friction in porous medium with magnetic field at $\frac{1}{k}+\frac{\sigma B_{0}^{2}}{\mu}=32$ is negative in the interval $0.4 \leq y \leq 0.6$.

Again by the graph of table- 4 of equation (6), the skin friction in porous medium at $\frac{1}{k}=11$ is positive \& increases in the interval $0 \leq y \leq 0.6$ while skin friction in magnetic field at $\frac{\sigma B_{0}^{2}}{\mu}=21$ is negative excepts only at $y=.5$. The skin friction in porous medium with magnetic field at $\frac{1}{k}+\frac{\sigma B_{0}^{2}}{\mu}=32$ in the interval $0 \leq y \leq 0.3$ is positive while the skin friction in porous medium with magnetic field at $\frac{1}{k}+\frac{\sigma B_{0}^{2}}{\mu}=32$ is negative in the interval $0.4 \leq y \leq 0.6$.

Again by the graph of table- 6 of equation (6), the skin friction in magnetic field at $\frac{\sigma B_{0}^{2}}{\mu}=11$ is positive \& increases in the interval $0 \leq y \leq 0.6$ while the skin friction in porous medium at $\frac{1}{k}=21$ is negative excepts only at $y=.5$. The skin friction in porous medium with magnetic field at $\frac{1}{k}+\frac{\sigma B_{0}^{2}}{\mu}=32$ in the interval $0 \leq y \leq 0.3$ is positive while the skin friction in porous medium with magnetic field at $\frac{1}{k}+\frac{\sigma B_{0}^{2}}{\mu}=32$ is negative in the interval $0.4 \leq y \leq 0.6$.

Also we have investigated skin frictions, average velocity, the volumetric flow, drag coefficients \& stream lines by the equations (7), (8), (9), (10), (11), (12), (13) \& (14) respectively. 


\section{REFERENCES}

1. Aydin M, Fenner RT. Boundary element analysis of driven cavity flow for low and moderate Reynolds numbers. International Journal for Numerical Methods in Fluids. 2001 Sep 15; 37 (1):45-64.

2. Bahloul A, Boutana N, Vasseur P. Doublediffusive and Soret-induced convection in a shallow horizontal porous layer. Journal of Fluid Mechanics. 2003 Sep 1; 491:325.

3. Barbu V, Havârneanu T, Popa C, Sritharan SS. Exact controllability for the magneto hydrodynamic equations. Communications on Pure and Applied Mathematics: A Journal Issued by the Courant Institute of Mathematical Sciences. 2003 Jun; 56 (6):732-83.

4. Barkley D, Tuckerman LS. Stability analysis of perturbed plane Couette flow. Physics of Fluids. 1999 May; 11 (5):1187-95.

5. Barkley D, Tuckerman LS. Turbulent-laminar patterns in plane Couette flow in IUTAM Symposium on Laminar-Turbulent Transition and Finite Amplitude Solutions 2005 (pp. 107-127). Springer, Dordrecht.

6. Barkley D, Tuckerman LS. Mean flow of turbulentlaminar patterns in plane Couette flow. arXiv preprint physics/0701235. 2007 Jan 20.

7. Barragy E, Carey GF. Stream function-vorticity driven cavity solution using $\mathrm{p}$ finite elements. Computers \& Fluids. 1997 Jun 1; 26 (5):453-68.

8. Batchelor GK. A proposal concerning laminar wakes behind bluff bodies at large Reynolds number. Journal of fluid mechanics. 1956 Oct; 1 (4):388-98.

9. Batchelor GK. On steady laminar flow with closed streamlines at large Reynolds number. Journal of Fluid Mechanics. 1956 Jul; 1 (2):177-90.

10. Baytas AC, Pop I. Free convection in oblique enclosures filled with a porous medium. International Journal of Heat and Mass Transfer. 1999 Mar 1; 42 (6):1047-57.

11. Beam RM, Warming R. An implicit factored scheme for the compressible Navier-Stokes equations. AIAA journal. 1978 Apr; 16 (4):393402.

12. Beant S, Chanpreet S. Analysis of Vortex Motion in Porous Media. Journal of Electronics Cooling and Thermal Control. 2012 Jun 18; 2012.

13. Šulc R, Ditl P. Local energy dissipation rate in an agitated vessel-a comparison of evaluation methods. Fluid Dynamics. 2018 Mar 1; 53 (2):20011.

14. Šulc R, Ditl P, Jašíkova D, Kotek M, Kopecký V, Kysela B. Effect of Particle Image Velocimetry Setting Parameters on Local Velocity Measurements in an Agitated Vessel. Chemical Engineering \& Technology. 2019 Apr; 42 (4):82734.

15. Tsypkin GG, Shargatov VA. Influence of capillary pressure gradient on connectivity of flow through a porous medium. International Journal of Heat and Mass Transfer. 2018 Dec 1; 127:1053-63.

16. Gülhan A, Thiele T, Siebe F, Kronen R, Schleutker T. Aerothermal measurements from the ExoMars Schiaparelli capsule entry. Journal of Spacecraft and Rockets. 2019 Jan; 5 6(1):68-81.

\section{Author Acknowledgement:}

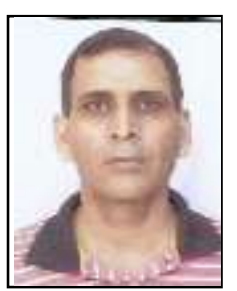

Dr. Anand Swrup Sharma is professor of Applied Sciences (Mathematics) at the Future University, Bareilly (India). He has a keen interest in fluid Mechanics. My Academic Qualification is M.Sc, M.Phil, \& PhD in Mathematics \& D.Sc (Scholar)

Email: $\underline{\text { sharma.as09@gmail.com }}$ 